\title{
Educación en tiempos de pandemia: tecnologías digitales en la mejora de los procesos educativos
}

\section{Education in Times of Pandemic: digital technologies for the improvement of educational processes}

\section{Educação em tempos de pandemia: tecnologias digitais na melhoria dos processos educacionais}

\begin{abstract}
Resumen: Las tecnologías digitales en el contexto de la pandemia COVID-19 han tomado relevancia al constituir, en muchos casos, el único nexo de los alumnos con las escuelas, trasladando los procesos de enseñanza al ámbito virtual. Al margen del enorme esfuerzo de muchos docentes, han quedado de manifiesto deficiencias crónicas del sistema educativo, entre ellas la baja competencia digital y, sobre todo, el aumento de la desigualdad en el acceso. Todo ello implica la necesidad de cambios en el sistema educativo en el tema digital -entre otrosy aparecen distintos interrogantes: ¿Qué tipo de respuesta damos a los problemas sobrevenidos? ¿Seguimos poniendo parches o aprovechamos la oportunidad para una verdadera transformación del sistema educativo? Estamos hablando de una oportunidad más para el rediseño radical de la enseñanza y la reinvención de la carrera docente que incorpore el desarrollo de la competencia digital.

Palabras clave: competencia digital, sistema educativo, entorno digital, carrera docente, COVID-19

Abstract: As a result of having to bring learning processes to a virtual environment, digital technologies in the COVID-19 pandemic context have become a relevant element for they are, in many cases, the sole nexus between students and their schools. Despite the enormous amount of effort by many of the teachers, particular chronic deficiencies of the educational system have been revealed. Among them: poor digital skills, and above all, an increasing inequality in access to these technologies. All that implies the need for a change in the educational system regarding digital technologies, which leads to the following questions: What kind of response have we been able to provide preceding these events? Are we still applying patches or are we making the best of this situation towards a true transformation of the educational system? The discussion here is about using this opportunity for making a radical redesign of teaching practices and for the reinvention of the teaching career with the incorporation of digital competence development.

Key Words: digital competence, educational system, digital environment, teaching career, COVID-19

Resumo: As tecnologias digitais no contexto da pandemia COVID-19 tomaram relevância ao constituir, em muitos casos, a única ligação dos estudantes com as escolas, deslocando os processos de ensino ao ambiente virtual. Para além do enorme esforço de muitos professores, as deficiências crônicas do sistema educacional, entre elas a baixa competência digital e, acima de tudo, o aumento da desigualdade de acesso. Tudo isto implica a necessidade de mudanças no sistema educacional na área digital -entre outras- e surgem várias questões: Que tipo de resposta damos aos problemas que surgiram? Continuamos remendando ou aproveitamos a oportunidade para uma verdadeira transformação do sistema educacional? Estamos falando de mais uma oportunidade para a reformulação radical do ensino e a reinvenção da carreira docente que incorpora o desenvolvimento da competência digital.
\end{abstract}

Palavras-chave: Competência digital, sistema educacional, ambiente digital, carreira docente, COVID-19 
En estos pocos meses se ha escrito -y, sobre todo, se ha hablado- de educación y COVID-19. La pandemia ha puesto a prueba las fortalezas del sistema educativo. Uno de los sectores donde los efectos de la pandemia han sido y siguen siendo más evidentes es la educación. En el pico de la pandemia, hasta 1.500 millones de estudiantes no asistieron a la escuela y en muchos casos todavía siguen sin hacerlo (UNESCO, 2020). Se trato de una medida abrupta y sin precedentes, planteando el desafío de proporcionar una vía segura y rápida de brindar aprendizaje desde casa. A pesar de todo, al menos una tercera parte de los niños en edad escolar de todo el mundo (463 millones) no tuvo acceso a la educación a distancia cuando la COVID-19 obligó a cerrar sus escuelas (UNICEF, 2020). Este informe revela las limitaciones de la educación a distancia y las graves desigualdades en el acceso.

Profesores y centros procuraron hacer frente a los estragos del confinamiento desde el compromiso y la responsabilidad. Eso sí, con una diversiad de respuestas, desde los que se adaptaron a la situación manteniendo propuestas de aprendizaje que situaban a los alumnos y sus familias en el centro de preocupación, generalmente centros que estaban ya inmersos en procesos de innovación y de transfromación hacia una mayor flexibilidad curricular y organizativa, hasta aquellos más tradicionales que intentaron trasladar el modelo tradicional presencial al ámbito virtual,obviamente con poco éxito.

El aprendizaje remoto se convirtió en la solución rápida. Pero esta enseñanza remota en emergencia, que utiliza de tecnologías digitales para hacer lo mismo que se hacía en presencial trasladando los procesos de enseñanza casi de forma arrebatada a través de tecnologías, realmente no corresponde a un sistema de aprendizaje online o por lo menos no responde a un modelo de aprendizaje o de enseñanzaaprendizaje online. En este contexto, es difícil que el docente pueda garantizar los tres tipos de presencia en la formación virtual, que sugieren Garrison y Anderson (2005): cognitiva, social y didáctica. Aquí se ha evidenciado la baja competencia digital mostrada por algunos docentes para la incorporación educativa de las tecnologías.

Es verdad que la crisis ha generado gran cantidad de respuestas impresionantes por parte de muchísimos educadores que han dado un paso al frente para servir a sus comunidades. También, que algunos sistemas de educación nacionales han podido ofrecer rápidamente experiencias de aprendizaje remoto. Pero la mayoría ha tenido problemas para satisfacer las necesidades de todos. Los usos de la tecnología educativa han resuelto en gran parte la situación, pero también han evidenciado deficiencias en algunos discursos muy asumidos: se ha tenido que regresar a modos comunicativos un poco más antiguos o asentados que se daban por superados, como ha ocurrido p.e. con el móvil erigido en el rey indiscutible, pero que en estas circunstancias ha tenldo que compaginarse con la radio y la televisión, por ejemplo.

Pero lo más grave ha sido que se ha resentido la equidad, el acceso y la capacidad para una educación inclusiva y de calidad. Este y otros problemas, críticos ya antes de la pandemia, se han manifestado con toda su crudeza, evidenciando la necesidad de cambios fundamentales. Se nos presenta una situación compleja que no podemos ignorar. ¿Qué tipo de respuesta damos a los problemas sobrevenidos? ¿Seguimos poniendo parches o aprovechamos la oportunidad para una verdadera transformación del sistema educativo?

Planificar en escenarios de incertidumbre resulta difícil: el ritmo de cambio de los escenarios es inferior al tiempo para profundizar en el análisis y, en consecuencia, es más fácil cometer errores, pero, al mismo tiempo, surge la oportunidad de gestionar de forma creativa los problemas inmediatos mientras construimos un puente hacia un sistema educativo reinventado.

El futuro de la educación se suele imaginar conectado, cada persona con su dispositivo con el que comunicar y acceder a toda la información. Es imaginado asociado a nuevas aplicaciones de la tecnología: realidad virtual o realidad aumentada utilizadas en algunos aprendizajes; tecnología en un aprendizaje 
cada vez más adaptativo; ubicuidad creciente de tecnologías portables -smartphones, tablets, etc.-; internet de las cosas y otras tecnologías digitales que incrementan su protagonismo en la comunicación educativa. Deberá enriquecerse, además, con estudios y contribuciones de diversos campos: trabajos recientes en neurociencias, en nanotecnología o en biotecnología; avances en psicología cognitiva sobre la inteligencia; consecuencias para el aprendizaje de las tecnologías, de las distintas formas de navegación virtual y procesamiento de la información, y otras que en este momento ni siquiera imaginamos.

Todo esto, aunque adquiera importancia en los años próximos, no parece que vaya a solucionar los problemas de aprendizaje que se dan en nuestras sociedades. El futuro es difícil de predecir, aunque sí que podemos participar en su construcción. Y contribuimos a construir este futuro de la educación construyendo una educación donde en lugar de certezas se trabaje con competencias digitales, con estrategias para enfrentarse a la incertidumbre, con la capacidad para resolver problemas nuevos. Así pues, un trabajo de prospectiva riguroso, que contribuya de verdad a tomar decisiones en la política educativa, tiene que bosquejar alguno de los escenarios futuros y la evolución que desde la realidad actual puede conducir a ellos.

La educación no puede disociarse de la escuela y del hecho de que docentes, alumnas y alumnos ya son ciudadanos digitales que viven en la sociedad del conocimiento. Por ello, uno de los primeros cambios hace referencia al rígido uso del tiempo y de la disposición espacial de las organizaciones educativas tal como las conocemos. Van a cambiar con toda seguridad, en combinación con un uso más cotidiano de las tecnologías digitales, y eso nos llevará a pensar e imaginar una nueva organización de los tiempos y de los espacios para las escuelas y centros educativos, diferentes de la actual disposición pensada para la era industrial. En el futuro, van a tener que ser flexibles y probablemente reflejarán mucho más lo que se vive en casa, en el trabajo y en la comunidad. El reto no parece ser la adopción de las tecnologías digitales en la vida escolar cotidiana, sino, la manera de promover la interacción humana, la sociabilidad y la colaboración en un mundo dominado por las frías tecnologías de la comunicación. Urge, entonces, crear escenarios educativos innovadores.

También requerirá atención la onmipresencia de la tecnología, de internet y de los distintos entornos digitales. Aquí, resulta útil hablar de competencia digital y su relación con el saber, que supone instaurar nuevas formas de pensar y trabajar en la escuela, supone construir un conocimiento que va más allá del mero acceso a la información y que se inscriba en trayectorias personales. El saber, más que información con utilidad inmediata, es una forma de apropiación del mundo y ésta, a partir de ahora, va a depender de la competencia digital de todos los actores.

Pero en rigor, el problema para la educación será el propio sentido de la institución escolar. ¿Para qué nos sirve la escuela en las sociedades contemporáneas? Tenemos ciertas dificultades para encontrar nuevas respuestas, y las del pasado ya no sirven. Las funciones clásicas atribuidas a la escuela de aprendizaje, socialización y custodia, cada vez presentan mayores contradicciones poniendo en cuestión tensiones entre estas funciones fruto de la evolución de la sociedad. Cuando la respuesta a estas tensiones son propuestas parciales, como señala Peña-López (2020) “rápidamente se deslizan por la pendiente de los ejes del privilegio, la dominación y la opresión, como bien se ha demostrado al considerar el enorme potencial emancipador o de exclusión (según la posición de quien lo mire) de la digitalización, la socialización o la segregación y la conciliación o el problema de la custodia". De nuestra respuesta a estas tensiones dependerá el futuro de la educación.

El Centre for Educational Research and Innovation (CERI) de la OCDE (2006), propuso ya seis escenarios posibles para la escuela del mañana que los agrupó en 3 tendencias: mantenimiento del status quo que incluiría tanto el escenario del 'mantenimiento de sistemas cada vez más burocráticos de enseñanza', como el del 'éxodo de profesores y desintegración del sistema'; una tendencia de evolución o de reescolarización, con dos escenarios: 'la escuela en el centro de la colectividad', y 'la escuela como organización 
centrada en el aprendizaje'; y una tendencia involucionista o de desescolarización, con dos escenarios posibles: 'expansión del modelo de mercado' y 'redes de educandos y sociedad en red'.

Novoa (2009), apunta a dos de estos escenarios que ayudan a reflexionar sobre el futuro de la educación. El primero -la escuela en el centro de la colectividad-insiste en las tendencias a la sobrecarga de la institución escolar de funciones sociales y culturales, a su papel asistencial, orientándola hacia tareas sociales de apoyo a los niños y niñas y a sus familias, sobre todo en el caso de los menos favorecidos $y$, sin dejar de lado la transmisión del saber, constituirse en lugar de referencia para las comunidades locales. El segundo -la escuela como organización centrada en el aprendizaje- destaca la importancia del saber y del aprendizaje en las sociedades del siglo XXI, rechazando la idea de que la escuela puede cargar con todo, pero identificando, en contrapartida, los aspectos centrales, específicos y prioritarios del trabajo escolar.

Se trata de un debate que seguro marca el futuro de la escuela y de la enseñanza ¿Resulta esencial evolucionar hacia una mayor responsabilización de la sociedad en temas educativos? Esto requiere la construcción de un espacio público de educación en el cual la escuela tenga su lugar, aunque no sea hegemónico. ¿Frente a las ideologías de la educación como un «bien privado», algunas particularmente seductoras (el home-schooling, p.e.), la educación deberá definirse como un «bien público»? Si es así, se refuerza una de las cualidades de la escuela pública: la posibilidad de instaurar historias compartidas y culturas de diálogo.

Los sistemas educativos se han de abrir a nuevas ideas: de la homogeneidad y la rigidez a la diferencia y el cambio; de la escuela sobrecargada a una nueva concepción del aprendizaje; del pasotismo de la sociedad, al refuerzo del espacio público de la educación.

También se han de abrir a nuevas formas de concebir la enseñanza. La investigación entendida como intervención integral, sistemática y basada en evidencias, pero también, y sobre todo, junto a la innovación y experimentación, debidamente evaluadas, deberán constituirse como procesos naturales en la construcción de los distintos escenarios (Castañeda, Salinas y Adell, 2020).

Estamos ante una oportunidad para el rediseño radical de la enseñanza y la reinvención de la carrera docente: Quienes se harán cargo de nuestra sociedad están entrando ya en la educación infantil; los nuevos docentes provienen ya de generaciones digitales. Superar una enseñanza preferentemente transmisiva requiere, tanto la transformación de un profesorado que tendrá que incorporar competencia digital docente, motivación y creatividad; como proyectos educativos colectivos innovadores que avancen hacia nuevas formas de enseñar, aprender, evaluar y vivir la educación.

Si en lugar de certezas trabajamos con competencias -las digitales entre ellas-, si la incertidumbre y la resolución de problemas nuevos los situamos en un lugar central, estaremos construyendo el futuro de la educación. 


\section{REFERENCIAS}

Castañeda, L., Salinas, J. y Adell, J. (2020). Hacia una visión contemporánea de la Tecnología Educativa. Digital Education Review, 37, 240-268 Recuperado de https://revistes.ub.edu/index.php/der/ article/view/30136

Garrison, D.R. y Anderson, T. (2005). El e-learning en el siglo XXI. Investigación y práctica. Barcelona: Octaedro.

OCDE (2006). Think Scenarios, Rethink Education. Paris: OECD Publishing. DOI: https://doi. org/10.1787/9789264023642-en.

Novoa, A. (2009). Educación 2021: para una historia del futuro. Revista lberoamericana de Educación, 49. Recuperado de https://rieoei.org/historico/documentos/rie49a07.htm

Peña, I. (2020). Los deberes atrasados del sistema educativo: digitalización, segregación y conciliación. CTXT, Contexto y Acción, 261. Recuperado de https://stecyl.net/ los-deberes-atrasados-del-sistema-educativo-digitalizacion-segregacion-y-conciliacion/

UNESCO (2020). Education: From disruption to recovery. Recuperado de https://en.unesco.org/covid19/ educationresponse

UNICEF (2020). COVID-19: Are children able to continue learning during school closures?. Recuperado de https://data.unicef.org/resources/remote-learning-reachability-factsheet/ 